\title{
A Bidirectional Subsethood Based Similarity Measure for Fuzzy Sets
}

\author{
Shaily Kabir*, Christian Wagner*, Timothy C. Havens ${ }^{\dagger}$, and Derek T. Anderson ${ }^{\ddagger}$ \\ *Intelligent Modelling and Analysis (IMA) Group and Lab for Uncertainty in Data and Decision Making (LUCID), \\ School of Computer Science, University of Nottingham, Nottingham, UK \\ ${ }^{\dagger}$ Dept. Electrical and Computer Engineering, Michigan Technological University, Houghton, MI, USA \\ ${ }^{\ddagger}$ Dept. Electrical Engineering and Computer Science, University of Missouri, Columbia, MO, USA \\ Email:\{shaily.kabir, christian.wagner\}@nottingham.ac.uk, thavens@mtu.edu, andersondt@missouri.edu
}

\begin{abstract}
Similarity measures are useful for reasoning about fuzzy sets. Hence, many classical set-theoretic similarity measures have been extended for comparing fuzzy sets. In previous work, a set-theoretic similarity measure considering the bidirectional subsethood for intervals was introduced. The measure addressed specific concerns of many common similarity measures, and it was shown to be bounded above and below by Jaccard and Dice measures respectively. Herein, we extend our prior measure from similarity on intervals to fuzzy sets. Specifically, we propose a vertical-slice extension where two fuzzy sets are compared based on their membership values. We show that the proposed extension maintains all common properties (i.e., reflexivity, symmetry, transitivity, and overlapping) of the original fuzzy similarity measure. We demonstrate and contrast its behaviour along with common fuzzy set-theoretic measures using different types of fuzzy sets (i.e., normal, non-normal, convex, and non-convex) in respect to different discretization levels.
\end{abstract}

\section{INTRODUCTION}

Similarity measures are important tools in fuzzy logic. Measures of similarity between two fuzzy sets capture how similar they are in terms of the degrees of membership of their elements. Generally, a similarity measure $S(A, B) \rightarrow[0,1]$ with fuzzy sets $A$ and $B$ in the universe of discourse $X$, satisfies the following properties [1]:

1) Reflexivity: $S(A, B)=1 \Longleftrightarrow A=B$;

2) Symmetry: $S(A, B)=S(B, A)$;

3) Transitivity: If $A \subseteq B \subseteq C$ then $S(A, B) \geq S(A, C)$;

4) Overlapping: If $A \cap B \neq \emptyset$ then $S(A, B)>0$. Otherwise, $S(A, B)=0$.

Many similarity measures have been proposed in the literature and among them, set-theoretic measures are well-known [2]. Commonly, classical set-theoretic similarity measures are extended to assess similarity between fuzzy sets [3], and a good overview of these measures can be seen in [2] and [4]. Among the existing fuzzy set-theoretic measures, the extended Jaccard similarity measure [5] is well-known and is often used for fuzzy sets. Two common approaches to extension are based on the vertical-slices and the $\alpha$-cuts [6] of fuzzy sets.

Recently, we have introduced a new set-theoretic similarity measure for (closed) intervals using their overlapping ratios [7] which is effectively equivalent to their respective degree of subsethood. In this paper, we thus use the more common term of 'subsethood' rather than 'overlapping ratio'. The new

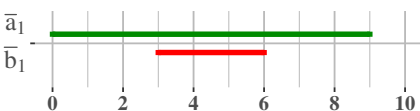

(a) Pair-1

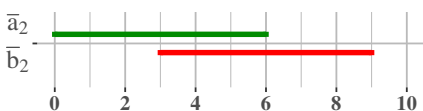

(b) Pair-2
Fig. 1. Two different example pairs of intervals

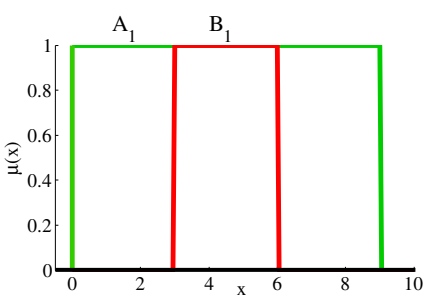

(a) Pair-1

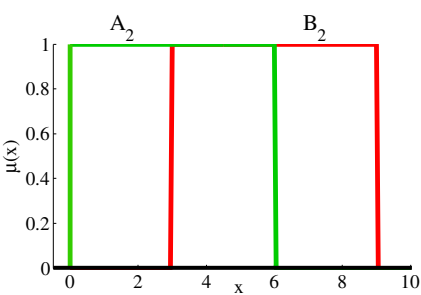

(b) Pair-2
Fig. 2. Fuzzy set representations for the pairs of intervals in Fig. 1

measure shows interesting results for certain features of the intervals - in particular, it is found to be more responsive to the size of the intervals and their intersection when one interval is a subset of the other. Further, it is bounded above and below by Jaccard and Dice measures respectively when the minimum t-norm is used. This finding inspires us to extend it for assessing similarity between fuzzy sets as both the vertical-slices and $\alpha$-cuts of the fuzzy set are generally (closed) intervals. For example, consider the interval pairs and their fuzzy set representations in Fig. 1 and Fig. 2. Here, both intervals and corresponding fuzzy set pairs are dissimilar-therefore intuitively should have a different degree of similarity. In [7], the new measure is shown to generate this expected difference in similarity, while both Jaccard and Dice measures show equal similarity. We expect that the extension of this new measure will also more appropriately capture the similarity between fuzzy sets with different degree of overlap, such as shown in Fig. 2, which is the core motivation of the extension proposed in this paper.

Herein, we propose an extension of the bidirectional subsethood based interval similarity measure [7] for type-1 (T1) fuzzy sets based on vertical slice decomposition as this approach is commonly applied for extending classical settheoretic measures. The $\alpha$-cut extension of this measure which compares fuzzy sets based on their universe of discourse will 
be discussed in context in an upcoming journal article. Here, following the extension, we explore major properties of the extended fuzzy measure. Later, we demonstrate the behaviour of the measure using different synthetic fuzzy sets (i.e. normal, non-normal, convex, and non-convex) and provide comparisons with existing fuzzy set-theoretic similarity measures.

The remainder of the paper is structured as follows. In Section II, we present some background on T1 fuzzy sets followed by a discussion on the subsethood and the bidirectional subsethood based interval similarity measure, and lastly, fuzzy set-theoretic similarity measures. Section III introduces the proposed vertical-slice extension of the bidirectional subsethood based measure for fuzzy sets and discusses its properties. A demonstration of the proposed extended measure and comparison with current fuzzy set-theoretic measures using synthetic fuzzy sets is provided in Section IV. Finally, Section V concludes the paper and hints at future work.

\section{BACKGROUND}

In this section, we first define T1 fuzzy sets and subsethood. Next, we discuss the bidirectional subsethood based similarity measure for intervals [7], followed by a brief overview of current fuzzy set-theoretic similarity measures.

\section{A. Type-1 fuzzy sets}

A T1 fuzzy set [8] is a set in which the membership of each element lies within the range of 0 and 1 . Typically, a fuzzy set in the universe of discourse $X$ is defined as:

$$
A=\left\{\left(x, \mu_{A}(x)\right) \mid x \in X, \mu_{A}(x) \in[0,1]\right\},
$$

where $\mu_{A}(x)$ is the membership grade of the element $x$ in the fuzzy set $A$ [9]. For a discrete universe of discourse, the fuzzy set $A$ is often written as

$$
A=\sum_{x} \mu_{A}(x) / x,
$$

where $\sum$ is the collection of all points $x \in X$ with membership value $\mu_{A}(x)$ [9].

A fuzzy set is normal if and only if $\sup _{x \in X} \mu_{A}(x)=1$. Along with, a fuzzy set is convex if and only if all of its $\alpha$ cuts are convex subsets of $X .{ }^{1}$ Note that an $\alpha$-cut of the fuzzy set $A$ is a non-fuzzy (crisp) set comprising all elements $x \in X$ whose membership grade within $A$ is greater than or equal to $\alpha$ [11], written as:

$$
\bar{A}_{\alpha}=\left\{x \mid \mu_{A}(x) \geq \alpha, \alpha \in[0,1]\right\} .
$$

\section{B. Subsethood}

Subsethood $S_{h}$ is a relation that indicates the degree to which one object is a subset of the other object. For two crisp sets, $a$ and $b, S_{h}$ is defined as follows [12]:

$$
S_{h}(a, b)=\frac{|a \cap b|}{|a|},
$$

\footnotetext{
${ }^{1} \mathrm{~A}$ set $C$ is convex if for any two points $c_{1}, c_{2} \in C$, the line segment $(1-\lambda) c_{1}+\lambda c_{2}, \lambda \in[0,1]$ lies in $C[10]$.
}

where $|a \cap b|$ is the cardinality of the intersection of $a$ and $b$, and $|a|$ is the cardinality of $a . S_{h}$ is bounded by $[0,1]$ where $S_{h}(a, b)=1$ means $a$ is a proper subset of $b(a \subseteq b)$, and $S_{h}(a, b)=0$ means $a$ is not a subset of $b(a \not \subset b)$.

As for intervals, the subsethood between two (closed) intervals $\bar{a}$ and $\bar{b}$ can be specified as:

$$
S_{h}(\bar{a}, \bar{b})=\frac{|\bar{a} \cap \bar{b}|}{|\bar{a}|}
$$

where $|\bar{a} \cap \bar{b}|$ is the size of the intersection between $\bar{a}$ and $\bar{b}$ and $|\bar{a}|$ is the size of $\bar{a}$.

In a similar manner, for two fuzzy sets, $A$ and $B$, the degree of subsethood [13] is:

$$
S_{h}(A, B)=\frac{\sum_{i=1}^{n} \min \left(\mu_{A}\left(x_{i}\right), \mu_{B}\left(x_{i}\right)\right)}{\sum_{i=1}^{n} \mu_{A}\left(x_{i}\right)},
$$

where $\sum_{i=1}^{n} \mu_{A}\left(x_{i}\right) \quad$ is the cardinality of $A$ and $\sum_{i=1}^{n} \min \left(\mu_{A}\left(x_{i}\right), \mu_{B}\left(x_{i}\right)\right)$ is the cardinality of the intersection of membership functions of $A$ and $B$ using the minimum t-norm.

\section{Bidirectional subsethood based similarity measure for in- tervals}

A new set-theoretic similarity measure for (closed) intervals was introduced in [7] which uses the overlapping ratios $(O R)$ of a pair of intervals for capturing their reciprocal similarity and later to determine the overall similarity with these ratios. Here we use the term 'subsethood' instead of 'overlapping ratio' as they are mathematically equivalent. Equation (4) presents this measure for two intervals $\bar{a}$ and $\bar{b}$ [7]:

$$
\begin{aligned}
S_{S_{h}}(\bar{a}, \bar{b}) & =\star(O R(\bar{a}, \bar{b}), \text { OR }(\bar{b}, \bar{a})) \\
& \equiv \star\left(S_{h}(\bar{a}, \bar{b}), S_{h}(\bar{b}, \bar{a})\right),
\end{aligned}
$$

where $\star$ is a $t$-norm and $S_{h}$ is the subsethood for the intervals. Using Eq. (2), we can rewrite Eq. (4) as:

$$
S_{S_{h}}(\bar{a}, \bar{b})=\star\left(\frac{|\bar{a} \cap \bar{b}|}{|\bar{a}|}, \frac{|\bar{a} \cap \bar{b}|}{|\bar{b}|}\right) .
$$

The bidirectional subsethood based similarity measure directly captures changes in the width of intervals and also to the size of their intersection when one interval is a subset of another in a pair. Further, it is bounded above and below by the well-known Jaccard and Dice measures in case of the minimum t-norm. Furthermore, it is invariant to multiplication of the interval endpoints and shows expected linear behaviour in respect of linearly increasing interval overlap.

In Section III, we introduce the vertical-slice extension of this measure for fuzzy sets where they are compared regarding the membership values and also discuss its properties. 
TABLE I

SET-THEORETIC SIMILARITY MEASURES AND THEIR PROPERTIES FOR FUZZY SETS $A$ AND $B$

\begin{tabular}{|c|l||c|c|c|c|}
\hline \multicolumn{2}{|c||}{ Similarity Measure } & \multicolumn{4}{c|}{ Properties } \\
\hline Equation & Proposed by & Reflexivity & Symmetry & Transitivity & Overlapping \\
\hline \hline$S_{J}(A, B)=\frac{\sum_{i=1}^{n} \min \left(\mu_{A}\left(x_{i}\right), \mu_{B}\left(x_{i}\right)\right)}{\sum_{i=1}^{n} \max \left(\mu_{A}\left(x_{i}\right), \mu_{B}\left(x_{i}\right)\right)}$ & $\begin{array}{l}\text { Pappis and } \\
\text { Karacapilidis [16] }\end{array}$ & Yes & Yes & Yes & $>0$ \\
\hline$S_{C}(A, B)=\max \left(\min \left(\mu_{A}\left(x_{i}\right), \mu_{B}\left(x_{i}\right)\right)\right.$ & Chen et al. [17] & No & Yes & No & $>0$ \\
\hline$S_{P}(A, B)=\frac{\sum_{i=1}^{n} \mu_{A}\left(x_{i}\right) \times \mu_{B}\left(x_{i}\right)}{\max \left(\sum_{i=1}^{n}\left(\mu_{A}\left(x_{i}\right)\right)^{2}, \sum_{i=1}^{n}\left(\mu_{B}\left(x_{i}\right)\right)^{2}\right)}$ & Chen et al. [17] & Yes & Yes & No & $>0$ \\
\hline$S_{W}(A, B)=\frac{1}{n} \sum_{i=1}^{n} \frac{\min \left(\mu_{A}\left(x_{i}\right), \mu_{B}\left(x_{i}\right)\right)}{\max \left(\mu_{A}\left(x_{i}\right), \mu_{B}\left(x_{i}\right)\right)}$ & Wang [18] & Yes & Yes & Yes & $>0$ \\
\hline$S_{R}(A, B)=\frac{\max \left(\min \left(\mu_{A}(x), \mu_{B}(x)\right)\right)}{\max \left(\max \left(\mu_{A}(x)\right), \max _{x}\left(\mu_{B}(x)\right)\right)}$ & Raha et al. [19] & Yes & Yes & Yes & $>0$ \\
\hline
\end{tabular}

\section{Fuzzy set-theoretic similarity measures}

Similarity assessment between two fuzzy sets using settheoretic measures typically involves fuzzy set operations, such as union, intersection, and cardinality. In most cases, classical set-theoretic similarity measures are extended to determine similarity for fuzzy sets [3]. It is worth noting that Tversky's parameterized ratio model [14] generalizes many classical set-theoretic similarity measures which expresses the similarity between two crisp sets $a$ and $b$ as a ratio of their common and distinct features:

$$
S_{T}(a, b)=\frac{f(a \cap b)}{f(a \cap b)+\alpha f(a-b)+\beta f(b-a)},
$$

where $f(a \cap b)$ presents the common features, $f(a-b)$ is the features that $a$ has but $b$ does not, and vice versa. The factors $\alpha$ and $\beta$ are nonnegative $(\geq 0) . S_{T}(a, b)$ turns into the Jaccard measure [5] when $\alpha=\beta=1$, whereas $S_{T}(a, b)$ becomes the Dice measure [15] with $\alpha=\beta=0.5$. For $\alpha=1$ and $\beta=0, S_{T}(a, b)$ behaves like the subsethood for $a$ (the degree to which $a$ is a subset of $b$ ).

Among the existing fuzzy set-theoretic similarity measures, the extended Jaccard measure [5] proposed by Pappis and Karacapilidis [16] is widely used for fuzzy sets. Besides, the fuzzy measures introduced by Chen et al. [17], Wang [18] and Raha et al. [19] are also well-known. In Table I, we summarize these similarity measures together with their essential properties. A fuzzy measure based on matching functions [17] is also included in Table I as it uses the maximum t-conorm. A detailed discussion of set-theoretic similarity measures for fuzzy sets can be found in [20] and [21].

\section{A NEW BIDIRECTIONAL SUBSETHOOD BASED SIMILARITY MEASURE FOR FUZZY SETS}

This section introduces a vertical-slice extension of the interval similarity measure $\left(S_{S_{h}}\right)$ [7] to assess similarity between two T1 fuzzy sets using the bidirectional subsethood.

We first present the proposed extension of the $S_{S_{h}}$ measure at Eq. (4) and then demonstrate its major properties.

\section{A. Proposed extension of the $S_{S_{h}}$ measure}

In the vertical-slice extension, we first determine the reciprocal subsethoods for a pair of fuzzy sets and then take a t-norm of these subsethood measure outcomes to determine the overall similarity between them. The proposed extension of the $S_{S_{h}}$ measure for fuzzy sets, $A$ and $B$, is defined as:

$$
S_{S_{h}}^{T 1}(A, B)=\star\left(S_{h}(A, B), S_{h}(B, A)\right) .
$$

Throughout this paper, we use the minimum and the product t-norms as they are used most often in fuzzy set theory. Using Eq. (3) for the subsethood $S_{h}$, we rewrite Eq. (6) as follows:

$S_{S_{h}}^{T 1}(A, B)=\star\left(\frac{\sum_{i=1}^{n} \min \left(\mu_{A}\left(x_{i}\right), \mu_{B}\left(x_{i}\right)\right)}{\sum_{i=1}^{n} \mu_{A}\left(x_{i}\right)}, \frac{\sum_{i=1}^{n} \min \left(\mu_{A}\left(x_{i}\right), \mu_{B}\left(x_{i}\right)\right)}{\sum_{i=1}^{n} \mu_{B}\left(x_{i}\right)}\right)$

where $n$ is the number of discretizations of the whole support of $A$ and $B .^{23}$

\section{B. Properties of the extended similarity measure}

This section introduces and proves the properties of the extended $S_{S_{h}}^{T 1}$ measure in Eq. (7) for fuzzy sets $A$ and $B$.

Theorem 1. (Boundedness). $S_{S_{h}}^{T 1}(A, B) \in[0,1]$.

Proof: The subsethood $S_{h}(A, B)$ is bounded by $[0,1]$ [13], which follows that the extended similarity measure $S_{S_{h}}^{T 1}(A, B)$ is also bounded by $[0,1]$.

Theorem 2. (Reflexivity). $S_{S_{h}}^{T 1}(A, B)=1 \Longleftrightarrow A=B$.

Proof: When $A=B, S_{h}(A, B)=S_{h}(B, A)=1$. From the boundary conditions of the $t$-norm $(\star)$ [22], $\star(1,1)=1$, thus making $S_{S_{h}}(A, B)=1$. Alternatively, $S_{S_{h}}(A, B)=1$

\footnotetext{
${ }^{2}$ We note that in case of discrete fuzzy sets $A$ and $B$ (where they have the same number of elements), $n$ denotes the number of elements in $A$ and $B$.

${ }^{3}$ We note that Eq. (7) behaves like a fuzzy measure mentioned in [21] for the minimum $t$-norm, though the origin of this measure is unknown.
} 
means that both $S_{h}(A, B)$ and $S_{h}(B, A)$ are equal to 1 . This only happens if $A$ and $B$ are identical.

Theorem 3. (Symmetry). $S_{S_{h}}^{T 1}(A, B)=S_{S_{h}}^{T 1}(B, A)$.

Proof: The t-norm $(\star)$ is symmetric [22]. Therefore, $S_{S_{h}}^{T 1}(A, B)$ is also symmetric.

Theorem 4. (Transitivity). $S_{S_{h}}^{T 1}(A, B) \geq S_{S_{h}}^{T 1}(A, C)$ when $A \subseteq B \subseteq C$.

Proof: When $A \subseteq B \subseteq C$, it follows that

$$
\sum_{x} \mu_{A}(x) \leq \sum_{x} \mu_{B}(x) \leq \sum_{x} \mu_{C}(x) .
$$

Case 1: when $\star$ is the minimum t-norm.

$$
\begin{aligned}
S_{S_{h}}^{T 1}(A, B) & =\star\left(\frac{\sum_{x} \min \left(\mu_{A}(x), \mu_{B}(x)\right)}{\sum_{x} \mu_{A}(x)}, \frac{\sum_{x} \min \left(\mu_{A}(x), \mu_{B}(x)\right)}{\sum_{x} \mu_{B}(x)}\right) \\
& =\frac{\sum_{x} \min \left(\mu_{A}(x), \mu_{B}(x)\right)}{\sum_{x} \mu_{B}(x)} \\
& \text { [from Eq. (8)] } \\
S_{S_{h}}^{T 1}(A, C) & =\star\left(\frac{\sum_{x} \min \left(\mu_{A}(x), \mu_{C}(x)\right)}{\sum_{x} \mu_{A}(x)}, \frac{\sum_{x} \min \left(\mu_{A}(x), \mu_{C}(x)\right)}{\sum_{x} \mu_{C}(x)}\right) \\
& =\frac{\sum_{x} \min \left(\mu_{A}(x), \mu_{C}(x)\right)}{\sum_{x} \mu_{C}(x)}
\end{aligned}
$$

From Eq. (8), $\sum_{x} \mu_{B}(x) \leq \sum_{x} \mu_{C}(x)$, thus making

$$
\frac{\sum_{x} \min \left(\mu_{A}(x), \mu_{B}(x)\right)}{\sum_{x} \mu_{B}(x)} \geq \frac{\sum_{x} \min \left(\mu_{A}(x), \mu_{C}(x)\right)}{\sum_{x} \mu_{C}(x)} .
$$

Hence $S_{S_{h}}^{T 1}(A, B) \geq S_{S_{h}}^{T 1}(A, C)$.

Case 2: when $\star$ is the product $t$-norm.

$$
\begin{aligned}
S_{S_{h}}^{T 1}(A, B)= & \star\left(\frac{\sum_{x} \min \left(\mu_{A}(x), \mu_{B}(x)\right)}{\sum_{x} \mu_{A}(x)}, \frac{\sum_{x} \min \left(\mu_{A}(x), \mu_{B}(x)\right)}{\sum_{x} \mu_{B}(x)}\right) \\
= & \frac{\sum_{x} \min \left(\mu_{A}(x), \mu_{B}(x)\right)^{2}}{\sum_{x} \mu_{A}(x) \times \sum_{x} \mu_{B}(x)} \\
S_{S_{h}}^{T 1}(A, C)= & \star\left(\frac{\sum_{x} \min \left(\mu_{A}(x), \mu_{C}(x)\right)}{\sum_{x} \mu_{A}(x)}, \frac{\sum_{x} \min \left(\mu_{A}(x), \mu_{C}(x)\right)}{\sum_{x} \mu_{C}(x)}\right) \\
= & \frac{\sum_{x} \min \left(\mu_{A}(x), \mu_{C}(x)\right)^{2}}{\sum_{x} \mu_{A}(x) \times \sum_{x} \mu_{C}(x)} \quad \text { [from Eq. (8)] }
\end{aligned}
$$

From Eq. (8), $\sum_{x} \mu_{B}(x) \leq \sum_{x} \mu_{C}(x)$. Hence,

$$
\frac{\sum_{x} \min \left(\mu_{A}(x), \mu_{B}(x)\right)}{\sum_{x} \mu_{B}(x)} \geq \frac{\sum_{x} \min \left(\mu_{A}(x), \mu_{C}(x)\right)}{\sum_{x} \mu_{C}(x)},
$$

It follows that

$$
\frac{\sum_{x} \min \left(\mu_{A}(x), \mu_{B}(x)\right)^{2}}{\sum_{x} \mu_{B}(x)} \geq \frac{\sum_{x} \min \left(\mu_{A}(x), \mu_{C}(x)\right)^{2}}{\sum_{x} \mu_{C}(x)},
$$

$$
\Longrightarrow \frac{\sum_{x} \min \left(\mu_{A}(x), \mu_{B}(x)\right)^{2}}{\sum_{x} \mu_{A}(x) \times \sum_{x} \mu_{B}(x)} \geq \frac{\sum_{x} \min \left(\mu_{A}(x), \mu_{C}(x)\right)^{2}}{\sum_{x} \mu_{A}(x) \times \sum_{x} \mu_{C}(x)} .
$$

Thus $S_{S_{h}}^{T 1}(A, B) \geq S_{S_{h}}^{T 1}(A, C)$.

Theorem 5. (Overlapping). $S_{S_{h}}^{T 1}(A, B)>0$ when $A \cap B \neq \emptyset$; otherwise, $S_{S_{h}}^{T 1}(A, B)=0$.

Proof: If $A \cap B \neq \emptyset, \exists x$ such that $\min \left(\mu_{A}(x), \mu_{B}(x)\right)>$ 0 , which follows that the numerator of Eq. (7) is

$$
\sum_{x} \min \left(\mu_{A}(x), \mu_{B}(x)\right)>0 .
$$

For the denominators in Eq. (7),

$$
\begin{gathered}
\sum_{x} \mu_{A}(x) \geq \sum_{x} \min \left(\mu_{A}(x), \mu_{B}(x)\right) \quad \text { and } \\
\sum_{x} \mu_{B}(x) \geq \sum_{x} \min \left(\mu_{A}(x), \mu_{B}(x)\right) .
\end{gathered}
$$

Therefore $S_{S_{h}}^{T 1}(A, B)>0$.

Conversely, if $A \cap B=\emptyset$, then $\min \left(\mu_{A}(x), \mu_{B}(x)\right)=0$ $\forall x$, thus making the numerator of Eq. (7) as

$$
\sum_{x} \min \left(\mu_{A}(x), \mu_{B}(x)\right)=0 .
$$

Therefore,

$$
\begin{gathered}
\frac{\sum_{x} \min \left(\mu_{A}(x), \mu_{B}(x)\right)}{\sum_{x} \mu_{A}(x)}=0 \text { and } \\
\frac{\sum_{x} \min \left(\mu_{A}(x), \mu_{B}(x)\right)}{\sum_{x} \mu_{B}(x)}=0
\end{gathered}
$$

Hence $S_{S_{h}}^{T 1}(A, B)=0$.

\section{Demonstrations}

This section presents the behaviour of the proposed extended measure (Eq. (6)) in comparison with some of the well-known fuzzy set-theoretic similarity measures using a key, but not exhaustive set of synthetic sample cases, focusing on the comparison of both normal/non-normal and convex/non-convex fuzzy sets. For the experiments, we apply both minimum and product $t$-norms for the proposed extension. Moreover, we use trapezoidal and Gaussian fuzzy sets as the trapezoidal membership function is simple yet captures a great deal of flexibility and can model antisymmetric concepts, and the Gaussian function is the engineers' favourite statistical representation. All experiments are implemented using Java in an Intel(R) Core(TM) i3-4005U series based machine running at $1.70 \mathrm{GHz}$ with $8 \mathrm{~GB}$ RAM.

We compare the $S_{S_{h}}^{T 1}$ measure at Eq. (6) with common fuzzy set-theoretic similarity measures (discussed in Table I) using synthetic fuzzy sets shown in Fig. 3 to Fig. 7. We use synthetic sets as they can generate interesting cases and do a bit more rigorous study. We demonstrate the behaviour of the similarity 


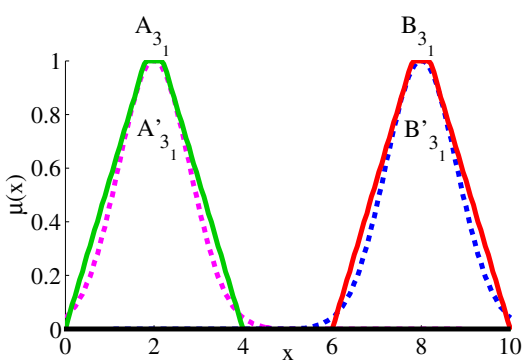

(a) Pair-1

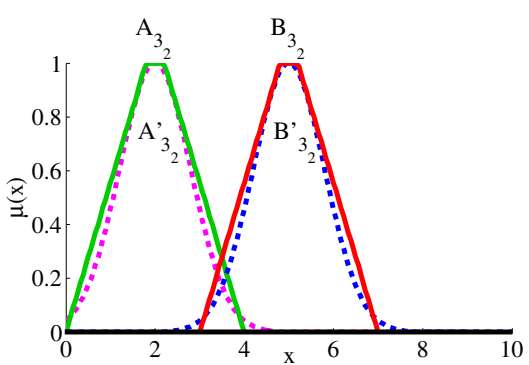

(b) Pair-2

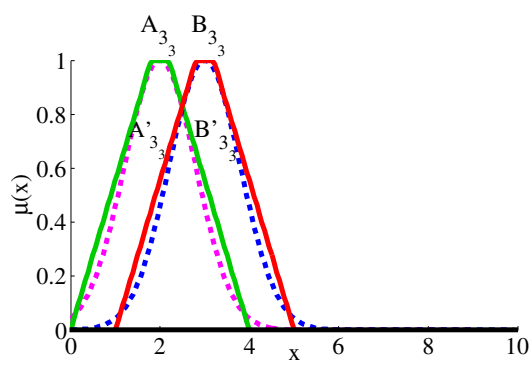

(c) Pair-3

Fig. 3. Trapezoidal (solid line) and Gaussian (dashed line) fuzzy sets with increasing overlap from left to right

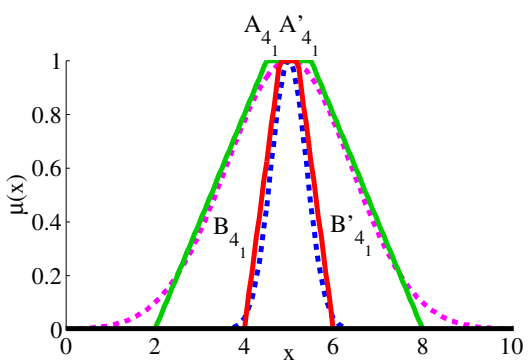

(a) Pair-1

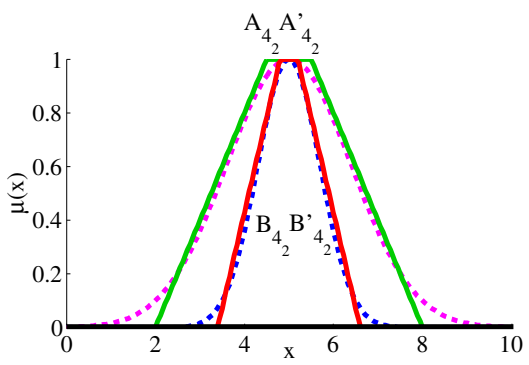

(b) Pair-2

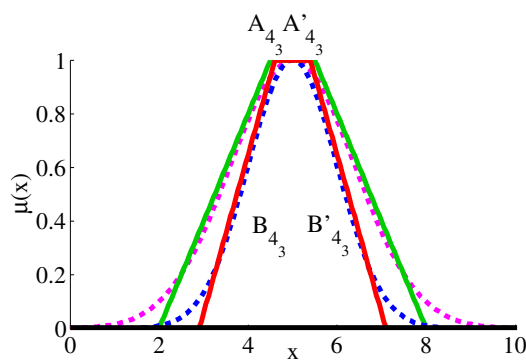

(c) Pair-3

Fig. 4. Trapezoidal (solid line) and Gaussian (dashed line) fuzzy sets with increasing degree of subsethood from left to right

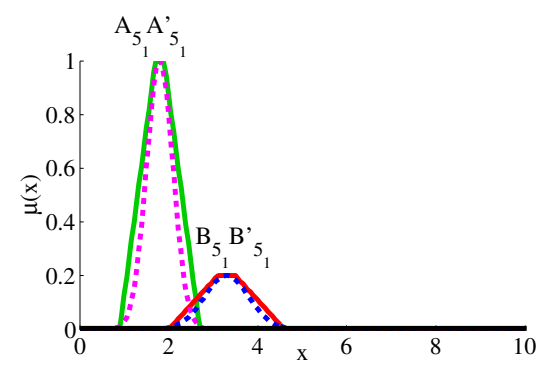

(a) Pair-1

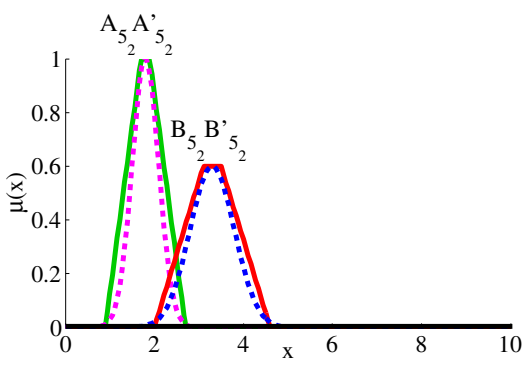

(b) Pair-2

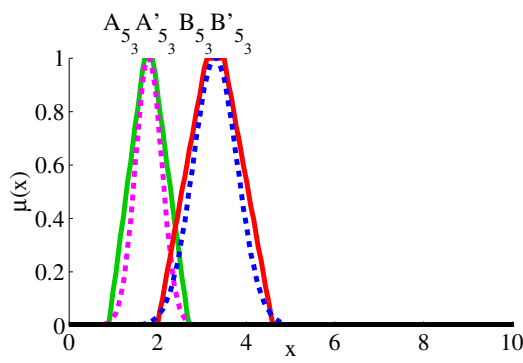

(c) Pair-3

Fig. 5. Trapezoidal (solid line) and Gaussian (dashed line) fuzzy sets with a rising height of non-normal fuzzy set from left to right while other set is normal

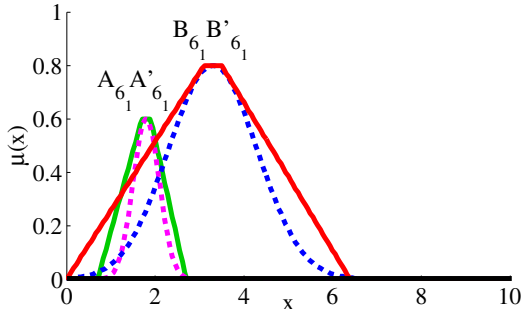

(a) Pair-1

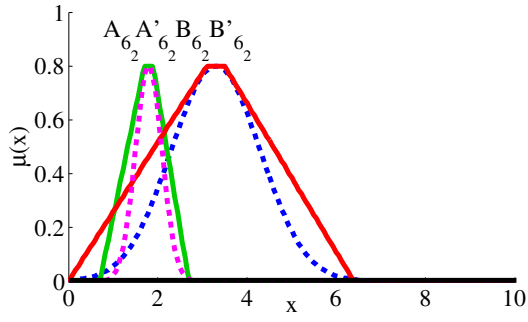

(b) Pair-2

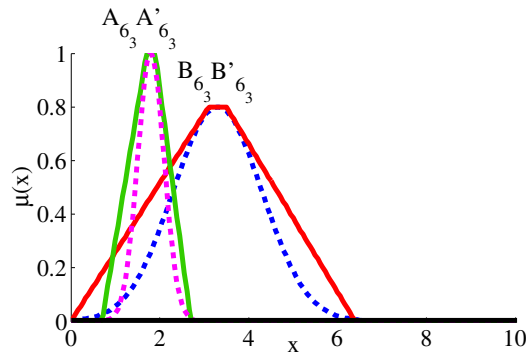

(c) Pair-3

Fig. 6. Trapezoidal (solid line) and Gaussian (dashed line) fuzzy sets with a rising height of non-normal fuzzy set from left to right while other set is non-normal

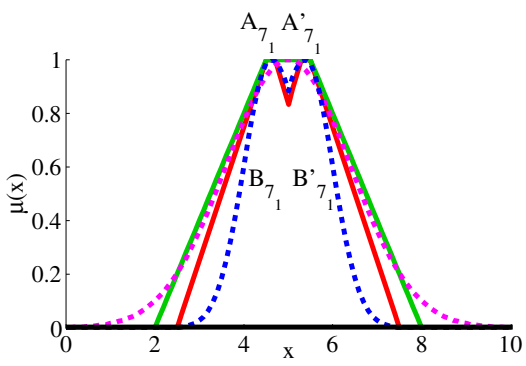

(a) Pair-1

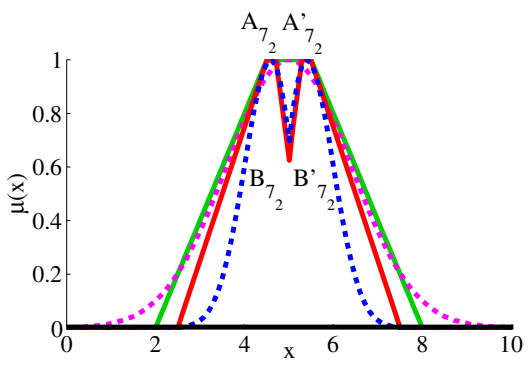

(b) Pair-2

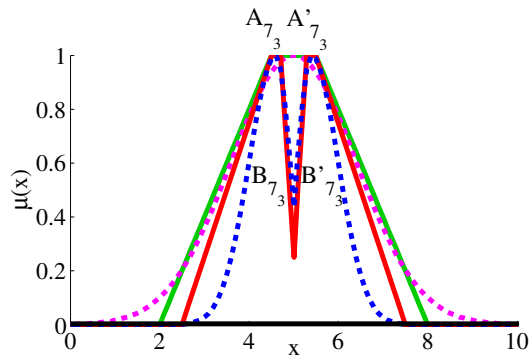

(c) Pair-3

Fig. 7. Trapezoidal (solid line) and Gaussian (dashed line) fuzzy sets with 'increasing non-convexity' from left to right 
TABLE II

SIMILARITY RESULTS FOR THE PAIRS OF TRAPEZOIDAL FUZZY SETS

\begin{tabular}{|c|c|c|c|c|c|c|c|c|c|c|c|c|c|c|c|}
\hline \multirow[b]{2}{*}{ Similarity Measure } & \multicolumn{3}{|c|}{$\begin{array}{l}\text { Increasing Overlap } \\
\text { (Fig. 3) }\end{array}$} & \multicolumn{3}{|c|}{$\begin{array}{l}\text { Increasing Subsethood } \\
\text { (Fig. 4) }\end{array}$} & \multicolumn{3}{|c|}{$\begin{array}{l}\text { Rising Height of } N N F S \text { with } \\
\text { a Normal Pair (Fig. 5) }\end{array}$} & \multicolumn{3}{|c|}{$\begin{array}{l}\text { Rising Height of } N N F S \text { with } \\
\text { a Non-normal Pair (Fig. 6) }\end{array}$} & \multicolumn{3}{|c|}{$\begin{array}{l}\text { 'Increasing Non-convexity' } \\
\text { (Fig. 7) }\end{array}$} \\
\hline & Pair-1 & Pair-2 & Pair-3 & Pair-1 & Pair-2 & Pair-3 & Pair-1 & Pair-2 & Pair-3 & Pair-1 & Pair-2 & Pair-3 & Pair-1 & Pair-2 & Pair-3 \\
\hline$S_{J}(A, B)$ & 0.0 & 0.0326 & 0.3968 & 0.3429 & 0.5143 & 0.7143 & 0.0313 & 0.0515 & 0.0541 & 0.2112 & 0.2219 & 0.224 & 0.8429 & 0.8245 & 0.7929 \\
\hline$S_{C}(A, B)$ & 0.0 & 0.2778 & 0.8333 & 1.0 & 1.0 & 1.0 & 0.1108 & 0.2658 & 0.3676 & 0.5178 & 0.5533 & 0.5764 & 1.0 & 1.0 & 1.0 \\
\hline$S_{P}(A, B)$ & 0.0 & 0.0322 & 0.724 & 0.4422 & 0.6275 & 0.817 & 0.0177 & 0.0532 & 0.0573 & 0.1925 & 0.2567 & 0.3209 & 0.906 & 0.883 & 0.841 \\
\hline$S_{W}(A, B)$ & 0.0 & 0.0552 & 0.2728 & 0.2042 & 0.3281 & 0.5001 & 0.0443 & 0.0662 & 0.0721 & 0.1991 & 0.1902 & 0.1767 & 0.6575 & 0.647 & 0.6282 \\
\hline$S_{R}(A, B)$ & 0.0 & 0.2778 & 0.8333 & 1.0 & 1.0 & 1.0 & 0.1108 & 0.2658 & 0.3676 & 0.6473 & 0.6916 & 0.5764 & 1.0 & 1.0 & 1.0 \\
\hline$S_{S_{h}: \min }^{T 1}(A, B)$ & 0.0 & 0.0631 & 0.5682 & 0.3429 & 0.5143 & 0.7143 & 0.0389 & 0.093 & 0.0961 & 0.2165 & 0.2406 & 0.2553 & 0.8429 & 0.8245 & 0.7929 \\
\hline$S_{S_{h}: \operatorname{prod}}^{T 1}(A, B)$ & 0.0 & 0.004 & 0.3228 & 0.3429 & 0.5143 & 0.7143 & 0.0051 & 0.0096 & 0.0111 & 0.1932 & 0.1789 & 0.1612 & 0.60 & 0.4571 & 0.40 \\
\hline
\end{tabular}

TABLE III

SIMILARITY RESULTS FOR THE PAIRS OF GAUSSIAN FUZZY SETS

\begin{tabular}{|c|c|c|c|c|c|c|c|c|c|c|c|c|c|c|c|}
\hline \multirow[b]{2}{*}{ Similarity Measure } & \multicolumn{3}{|c|}{$\begin{array}{c}\text { Increasing Overlap } \\
\text { (Fig. 3) }\end{array}$} & \multicolumn{3}{|c|}{$\begin{array}{l}\text { Increasing Subsethood } \\
\text { (Fig. 4) }\end{array}$} & \multicolumn{3}{|c|}{$\begin{array}{c}\text { Rising Height of NNFS with } \\
\text { a Normal Pair (Fig. 5) }\end{array}$} & \multicolumn{3}{|c|}{$\begin{array}{l}\text { Rising Height of } N N F S \text { with } \\
\text { a Non-normal Pair (Fig. 6) }\end{array}$} & \multicolumn{3}{|c|}{$\begin{array}{c}\text { 'Increasing Non-convexity' } \\
\text { (Fig. 7) }\end{array}$} \\
\hline & Pair-1 & Pair-2 & Pair-3 & Pair-1 & Pair-2 & Pair-3 & Pair-1 & Pair-2 & Pair-3 & Pair-1 & Pair-2 & Pair-3 & Pair-1 & Pair-2 & Pair-3 \\
\hline$S_{J}(A, B)$ & 0.0 & 0.0321 & 0.373 & 0.2858 & 0.5002 & 0.7145 & 0.0216 & 0.0304 & 0.0316 & 0.1282 & 0.1311 & 0.1325 & 0.4058 & 0.3647 & 0.3220 \\
\hline$S_{C}(A, B)$ & 0.0 & 0.1724 & 0.8226 & 1.0 & 1.0 & 1.0 & 0.0587 & 0.1249 & 0.1724 & 0.3835 & 0.4119 & 0.4322 & 0.9829 & 0.9773 & 0.9726 \\
\hline$S_{P}(A, B)$ & 0.0 & 0.0301 & 0.6766 & 0.3885 & 0.6325 & 0.822 & 0.0089 & 0.0268 & 0.0279 & 0.1099 & 0.1466 & 0.1832 & 0.5199 & 0.4619 & 0.4016 \\
\hline$S_{W}(A, B)$ & 0.0 & 0.0689 & 0.2929 & 0.1045 & 0.2024 & 0.3576 & 0.0389 & 0.0418 & 0.0434 & 0.1231 & 0.1167 & 0.1116 & 0.1639 & 0.1493 & 0.1342 \\
\hline$S_{R}(A, B)$ & 0.0 & 0.1724 & 0.8226 & 1.0 & 1.0 & 1.0 & 0.0587 & 0.1249 & 0.1724 & 0.4794 & 0.5148 & 0.4322 & 0.9829 & 0.9773 & 0.9726 \\
\hline$S_{S_{h}: \min }^{T 1}(A, B)$ & 0.0 & 0.0625 & 0.5426 & 0.2858 & 0.5002 & 0.7145 & 0.0281 & 0.0588 & 0.0625 & 0.1395 & 0.1521 & 0.1614 & 0.4062 & 0.3650 & 0.3222 \\
\hline$S_{S_{h}: p r o d}^{T 1}(A, B)$ & 0.0 & 0.0039 & 0.2949 & 0.2858 & 0.5002 & 0.7145 & 0.0024 & 0.0035 & 0.0042 & 0.0855 & 0.0762 & 0.0687 & 0.4022 & 0.3613 & 0.3186 \\
\hline
\end{tabular}

measures by focusing on the changes in similarity results while the properties of the fuzzy sets are gradually changing, i.e., by varying the degree of overlap, the subsethood, the height of (non-normal) fuzzy sets (NNFS), and their 'degree of non-convexity'. Table II and III present the similarity results considering 1000 discretization levels along the whole support of the pairs of trapezoidal and Gaussian fuzzy sets graphically shown in Fig. 3 to Fig. 7.

Convex-normal fuzzy sets - In Fig. 3, three pairs of trapezoidal $\left(A_{3}\right.$ and $\left.B_{3}\right)$ and Gaussian fuzzy sets $\left(A_{3}^{\prime}\right.$ and $\left.B_{3}^{\prime}\right)$ are used, where the degree of overlap between the pairs have been increased gradually. Here, all pairs are normal and convex. Specifically, pairs are shifted from completely disjoint to a gradually increasing overlap along the $\mathrm{x}$-axis. Intuitively, as the overlapping between pairs increases, similarity is expected to rise accordingly. Table II and III show expected behaviour of all similarity measures.

Convex-normal fuzzy sets (case for proper subsets) - In Fig. 4, three pairs of (normal and convex) trapezoidal $\left(A_{4}\right.$ and $\left.B_{4}\right)$ and Gaussian fuzzy sets $\left(A_{4}^{\prime}\right.$ and $\left.B_{4}^{\prime}\right)$ are considered, where $B_{4}\left(B_{4}^{\prime}\right)$ is a proper subset of $A_{4}\left(A_{4}^{\prime}\right)$. Here the degree of subsethood in the pairs is gradually increased. In this case, it is intuitive to expect that as the degree of subsethood gradually increases, similarity will also rise. Table II and III show that both $S_{C}$ and $S_{R}$ measures yield a similarity of 1 for all pairs despite being non-identical while the other measures return an increasing degree of similarity in line with expectation.

Convex fuzzy sets (case with one normal fuzzy set) - Three pairs of trapezoidal $\left(A_{5}\right.$ and $\left.B_{5}\right)$ and Gaussian fuzzy sets
( $A_{5}^{\prime}$ and $B_{5}^{\prime}$ ) are considered in Fig. 5 where $A_{5}$ and $A_{5}^{\prime}$ are normal while $B_{5}$ and $B_{5}^{\prime}$ are non-normal, and their heights are gradually increased. As the height of the non-normal fuzzy set increases, the similarity within the pairs should intuitively rise as their heights become more similar until reaching its maximum when both sets in a pair share the same height. Here, all measures maintain the expected rising trend in the similarity results.

Convex fuzzy sets (case with both fuzzy sets non-normal) For the pairs of fuzzy sets in Fig. 6 which are both convex and non-normal, all measures except $S_{R}$ behave non-intuitively by showing either the sets in Pair-1 or Pair-3 to be more similar than those in Pair-2. In this experiment, all fuzzy sets are initially non-normal, with $B_{6}$ and $B_{6}^{\prime}$ remaining non-normal, while $A_{6}$ and $A_{6}^{\prime}$ gradually turn into normal fuzzy sets.

Non-convex, normal fuzzy sets - Finally, three pairs of fuzzy sets - trapezoidal $\left(A_{7}\right.$ and $\left.B_{7}\right)$ and Gaussian $\left(A_{7}^{\prime}\right.$ and $\left.B_{7}^{\prime}\right)$ are considered in Fig. 7 where $B_{7}$ and $B_{7}^{\prime}$ are both non-convex fuzzy sets and proper subsets of $A_{7}$ and $A_{7}^{\prime}$ respectively. Here, the 'degree of non-convexity' is increased in a gradual manner. As the non-convexity 'increases', the degree of overlap, or, more formally, the cardinality of the intersection between the pairs declines, intuitively resulting in a decreasing degree of similarity. Here, both $S_{C}$ and $S_{R}$ measures show a perhaps unexpected behaviour for trapezoidal fuzzy sets - yielding a similarity of 1 for all pairs, while following the expected decreasing trend for the Gaussian fuzzy sets. All other measures show a decreasing trend for all pairs in respect to the similarity results. 
TABLE IV

EXECUTION TIME (ms) FOR DIFFERENT DISCRETIZATION LEVELS (250, $500,750,1000)$ ALONG THE WHOLE SUPPORT OF PAIR-1 OF THE GAUSSIAN FUZZY SETS $\left(A_{4_{1}}^{\prime}, B_{4_{1}}^{\prime}\right)$ IN FIG. 4(a)

\begin{tabular}{|c|c|c|c|c|}
\hline \multirow{2}{*}{ Similarity Measure } & \multicolumn{4}{|c|}{ No. of Discretization } \\
\cline { 2 - 5 } & 250 & 500 & 750 & 1000 \\
\hline \hline$S_{J}(A, B)$ & 17.43 & 29.62 & 36.28 & 41.86 \\
\hline$S_{C}(A, B)$ & 13.42 & 25.74 & 33.20 & 35.75 \\
\hline$S_{P}(A, B)$ & 15.40 & 27.40 & 34.08 & 41.37 \\
\hline$S_{W}(A, B)$ & 15.65 & 27.90 & 34.33 & 41.47 \\
\hline$S_{R}(A, B)$ & 14.50 & 26.48 & 33.25 & 38.40 \\
\hline$S_{S_{h}: \text { min }}^{T 1}(A, B)$ & 15.74 & 27.97 & 35.18 & 41.64 \\
\hline$S_{S_{h}^{T}: \text { prod }}^{T 1}(A, B)$ & 14.83 & 26.55 & 33.72 & 40.89 \\
\hline
\end{tabular}

In short, all measures show expected behaviour for varying degrees of overlap. Both $S_{C}$ and $S_{R}$ measures deviate from intuition while changing the degree of subsethood. For variations in the height of pairs of non-normal fuzzy sets (NNFS), only $S_{R}$ measure follows intuition. Finally, with a rising degree of non-convexity, both $S_{C}$ and $S_{R}$ measures show unexpected results for trapezoidal fuzzy sets while meeting the expectation for the Gaussian fuzzy sets. Overall, $S_{J}, S_{P}, S_{W}$ and the proposed extended $S_{S_{h}}^{T 1}$ (with minimum and product $t$ norms) measures largely follow intuition for a variety of fuzzy sets-except for pairs of fuzzy sets being non-normal. Initial work on developing an $\alpha$-cut based, rather than a verticalslice (as proposed here) extension of the original bidirectional subsethood measure indicates that this will result in a measure which also intuitively addresses such case of non-normal fuzzy sets. This will be explored as part of a future publication.

In addition, we provide respective execution times in milliseconds (ms) for various discretization levels $(250,500$, $750,1000)$ in Table IV for all measures. These have been empirically established taken by computing the similarity results for Pair-1 of the Gaussian fuzzy sets $\left(A_{4_{1}}^{\prime}, B_{4_{1}}^{\prime}\right)$ in Fig. 4(a). The results show that all measures share a similar execution time and we do not include further results as they follow the same trend.

\section{CONCLUSIONS}

In this paper, we have proposed a vertical-slice based extension of the recently introduced similarity measure [7] for T1 fuzzy sets (convex, non-convex and normal, non-normal). The proposed extension is based on the bidirectional subsethood of a pair of fuzzy sets. We have shown that it maintains all important properties of a similarity measure for fuzzy sets.

Further, we have demonstrated and compared the behavior of the extended measure with that of key existing fuzzy settheoretic similarity measures using a series of synthetic fuzzy sets. Here, the proposed extended measure shows expected behaviour in respect to variations of key properties such as an increase in the degree of overlap, subsethood, and height, or a change in the 'degree of non-convexity' of the fuzzy sets. However, $S_{C}$ and $S_{R}$ measures behave unexpectedly in cases of varying degrees of subsethood and non-convexity. Overall,
$S_{J}, S_{P}, S_{W}$, and the proposed extended $S_{S^{h}}^{T 1}$ (with minimum and product $t$-norms) measures largely meet the expectation for various types of fuzzy sets-except for the pairs where both are non-normal. Our initial work has shown that an $\alpha$ cut based extension of the bidirectional subsethood measure for intervals [7] can address this unexpected behaviour with fuzzy sets being non-normal. This, as well as the application of the new measure in aggregation and its extension to type-2 fuzzy sets will be explored as part of future publications.

\section{REFERENCES}

[1] J. McCulloch, C. Wagner, and U. Aickelin, "Analysing fuzzy sets through combining measures of similarity and distance," in Proc. IEEE Int. Conf. Fuzzy Systems. Beijing, China: IEEE, 2014, pp. 155-162.

[2] R. Zwick, E. Carlstein, and D. V. Budescu, "Measures of similarity among fuzzy concepts: A comparative analysis," International Journal of Approximate Reasoning, vol. 1, no. 2, pp. 221-242, 1987.

[3] D. Dubois and H. Prade, Fuzzy sets and systems: theory and applications. Academic press, Newyork, 1980

[4] L. Baccour, A. M. Alimi, and R. I. John, "Some notes on fuzzy similarity measures and application to classification of shapes recognition of arabic sentences and mosaic," IAENG International Journal of Computer Science, vol. 41, no. 2, pp. 81-90, 2014.

[5] P. Jaccard, "Nouvelles recherches sur la distribution florale," Bulletin de la Socit vaudoise des Sciences Naturelles, vol. 44, pp. 223-270, 1908.

[6] J. McCulloch, C. Wagner, and U. Aickelin, "Measuring the directional distance between fuzzy sets," in 13th UK Workshop on Computational Intelligence(UKCI). IEEE, 2013, pp. 38-45.

[7] S. Kabir, C. Wagner, T. C. Havens, D. T. Anderson, and U. Aickelin, "Novel similarity measure for interval-valued data based on overlapping ratio," in Proc. IEEE Int. Conf. Fuzzy Systems. Naples, Italy: IEEE, 2017.

[8] L. A. Zadeh, "Fuzzy sets," Information and control, vol. 8, no. 3, pp. 338-353, 1965.

[9] J. M. Mendel, Uncertain rule-based fuzzy logic systems: introduction and new directions. Prentice Hall PTR Upper Saddle River, 2001.

[10] H. S. Kasana and K. D. Kumar, Introductory Operations Research. Springer, 2004.

[11] L. A. Zadeh, "The concept of a linguistic variable and its application to approximate reasoning," Information sciences, vol. 8, no. 3, pp. 199249, 1975.

[12] H. T. Nguyen and K. Vladik, "Computing degrees of subsethood and similarity for interval-valued fuzzy sets: fast algorithms," in Proc. 9th Int. Conf. Intelligent Technologies (Intech'08), Thailand, 2008, pp. 4755.

[13] B. Kosko, "Fuzzy entropy and conditioning," Information Sciences, vol. 40, no. 2, pp. 165-174, 1986.

[14] A. Tversky, "Features of similarity." Psychological review, vol. 84, no. 4, p. $327,1977$.

[15] L. R. Dice, "Measures of the amount of ecologic association between species," Ecology, vol. 26, no. 3, pp. 297-302, 1945.

[16] C. P. Pappis and N. I. Karacapilidis, "A comparative assessment of measures of similarity of fuzzy values," Fuzzy Sets and Systems, vol. 56, no. 2, pp. 171-174, 1993.

[17] S.-M. Chen, M.-S. Yeh, and P.-Y. Hsiao, "A comparison of similarity measures of fuzzy values," Fuzzy sets and systems, vol. 72, no. 1, pp. 79-89, 1995.

[18] W.-J. Wang, "New similarity measures on fuzzy sets and on elements," Fuzzy sets and systems, vol. 85, no. 3, pp. 305-309, 1997.

[19] S. Raha, A. Hossain, and S. Ghosh, "Similarity based approximate reasoning: fuzzy contro," Journal of Applied Logic, vol. 6, no. 1, pp. 47-71, 2008.

[20] V. V. Cross and T. A. Sudkamp, Similarity and compatibility in fuzzy set theory: assessment and applications. Springer Science \& Business Media, 2002.

[21] A. Pal, B. Mondal, N. Bhattacharyya, and R. Swapan, "Similarity in fuzzy systems," Journal of Uncertainty Analysis and Applications, vol. 2, no. 18, pp. 1-28, 2014.

[22] D. M. Gabbay and J. Woods, Handbook of the History of Logic: The Many Valued and Nonmonotonic Turn in Logic. Elsevier, 2007. 